\title{
STRUCTURAL BRAIN PLASTICITY INDUCED BY PHYSICAL TRAINING IN ADULTS AFFECTED BY AGING OR DISEASE RELATED IMPAIRMENTS: A SYSTEMATIC REVIEU
}

\section{Jessica Van Oosterwijck *, Evy Dhondt *, Karen Caeyenberghs *^, Lieselot Burggraeve *, L.ieven Danneels *}

Ghent University, Belgium: * Department of Rehabilitation Sciences and Phusiotherapy, ^ Department of Movement and Sports Sciences

\section{BACKGROUND}

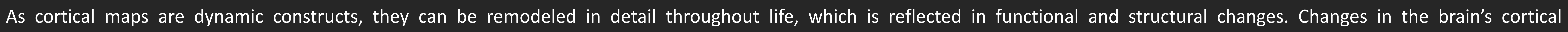
representation can occur as a consequence of alterations in input and behavior or as a consequence of aging and disease.

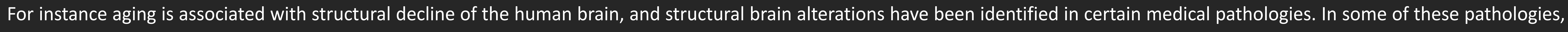

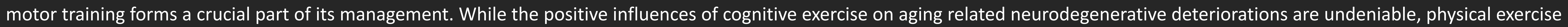
also has a positive impact on function, health status and quality of life in the elderly.

The question remains if structural plasticity of the brain underlies these beneficial effects of physical exercise in the these populations.

\section{AIMS}

To summarize the current evidence concerning the effect of physical therapy/training on the structure of the brain in elderly and adults affected by disease reflated impairments.

In addition, the existing evidence was examined to reveal whether exercise training induced changes in brain structure are associated with improvements in functional outcome.

\section{METHODS}

Following the PRISMA-guidelines a systematic search was performed in electronic search engines using a combination of predefined keywords regarding physical therapy/training and measures/outcomes of brain structure.

Full text reports from research studies assessing a structural component metric of the brain before and after a physical training intervention in humans which were geriatric or adults suffering from a medical condition were included.

The included articles were reviewed on methodological quality using the risk of bias tools provided by the Cochrane Centre.

\section{RESULTS}

From the 16 studies that were included, 5 studies (all RCT's) examined the effect of physical training in healthy elderly adults and 11 studies (7 RCT's, 4 case-controls) in patients suffering from medical conditions.

The most frequent causes for high risk of bias were failing to blind participants and therapist(s), not accounting for confounders, and incomplete data reporting.

The medical pathologies studied varied markedly, including neurological conditions, mental disorders, cognitive impairment, and musculoskeletal disorders.

Four groups of physical training could be roughly distinguished:

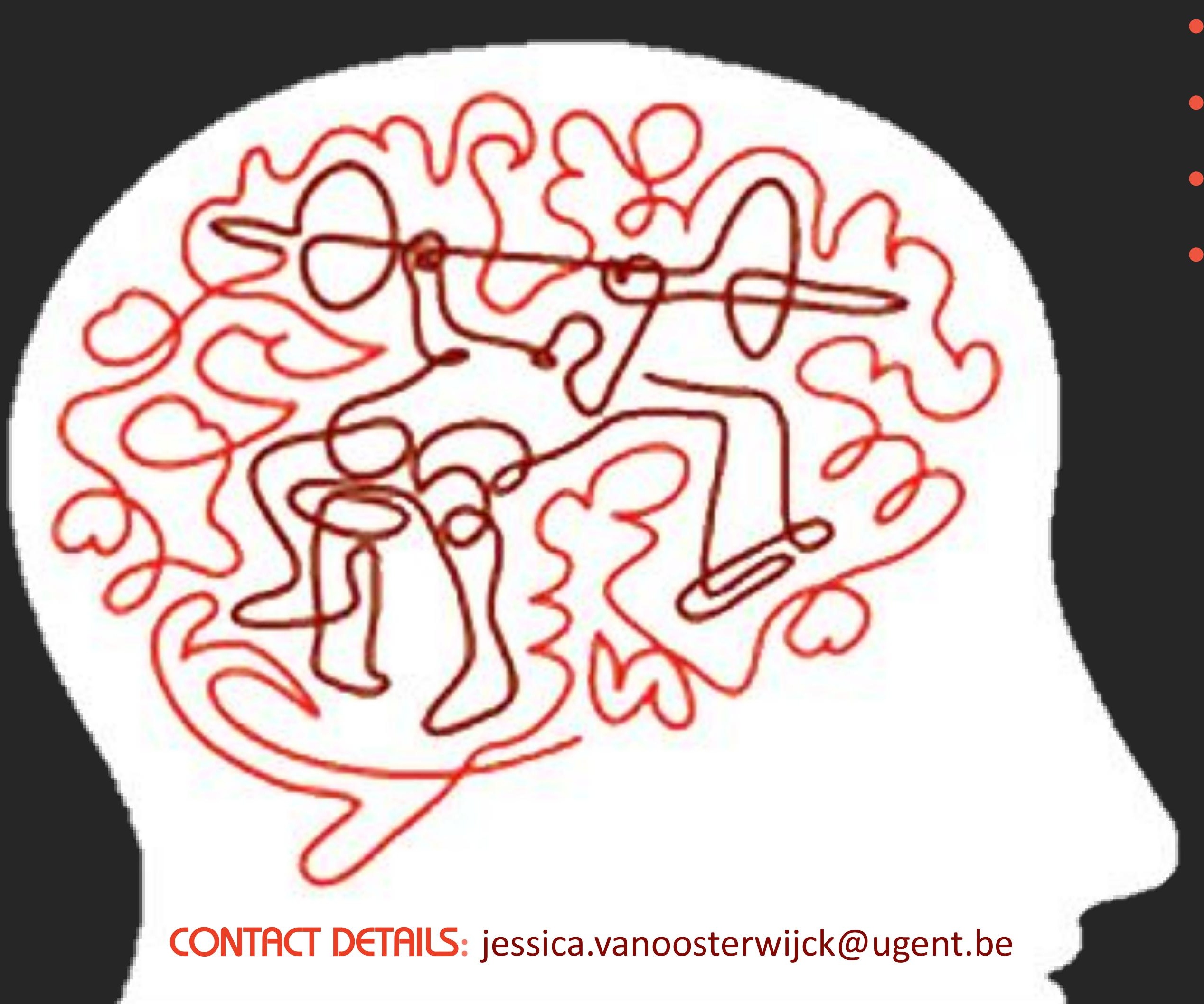

global exercise programs aerobic exercise programs

resistance training pathology-specific training

\section{$\widehat{\overline{\text { IIIIIII }}}$ UNIVERSITEIT GENT}

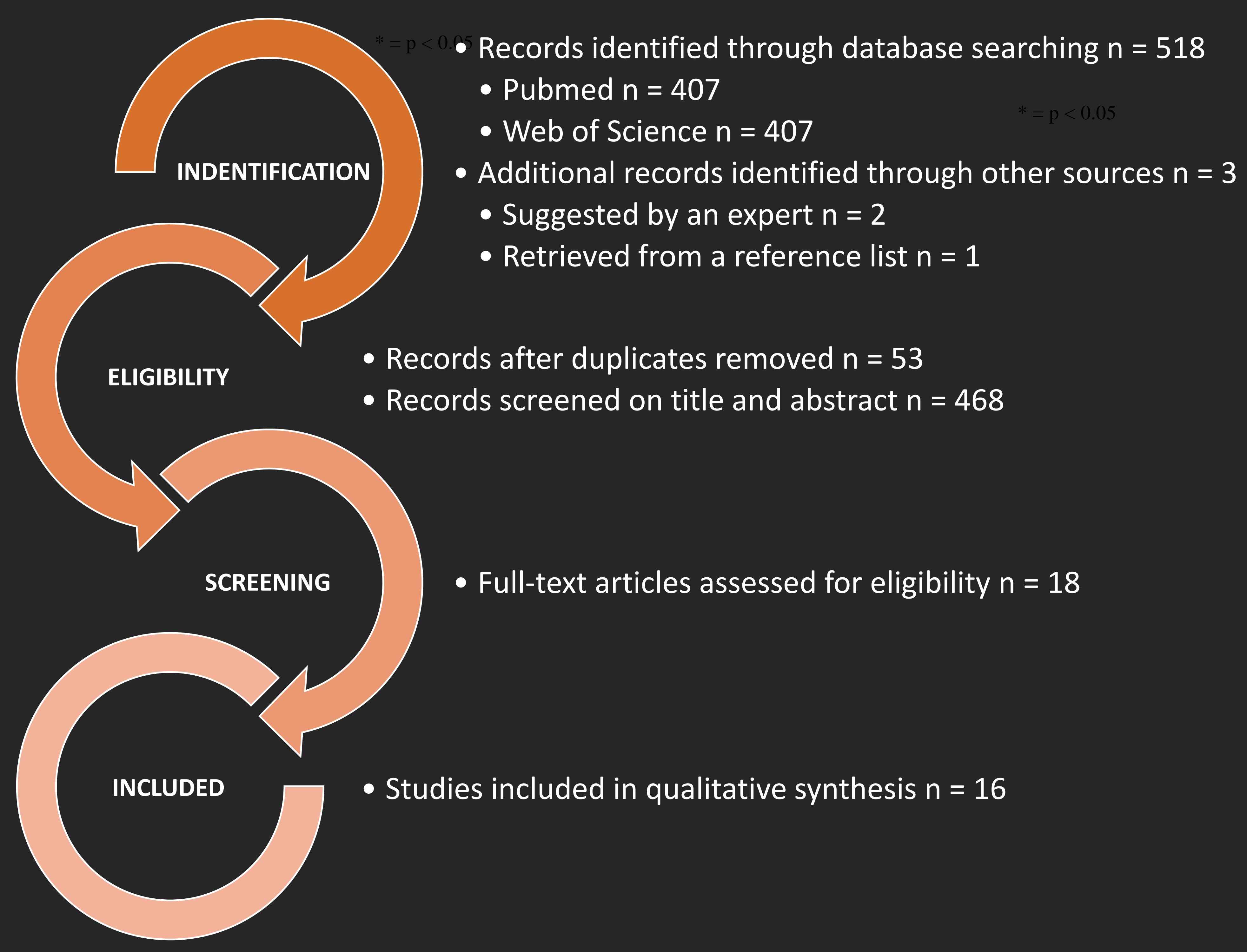

\section{DISCUSSION \& CONCLUSIONS}

\section{Physical training induced changes in structural brain plasticity}

There is strong evidence for increased gray matter volume in prefrontal cortex, temporal lobe (hippocampus) and supplementary motor area after aerobic training, and moderate evidence for increased gray matter volume in sensory \& motor cortices and temporal lobe (hippocampus) after pathology-specific training.

General exercise programs and resistance training do not increase gray matter volume.

There is weak evidence supporting cortical thickness increases after pathology specific training, but not following aerobic and general exercise programs.

In healthy elderly, aerobic exercise is neuroprotective and increases both gray and white mater volume.

Regarding white matter microstructural reorganization no clear conclusions can be drawn, due to the small amount of studies, different outcome reporting, and conflicting results.

\section{Association between training-related clinical changes and brain structural changes}

The observed volumetric brain changes, are related to post-training improvements in physical or cognitive performance. This indicates improvements in functionality due to training are subserved by structural brain plasticity. 